\title{
Mild balanoposthitis
}

\author{
C Veller Fornasa, A Calabro, A Miglietta, M Tarantello, C Biasinutto, A Peserico
}

\begin{abstract}
Aim-To identify and study cases of mild balanoposthitis (MBP) with penile pathology among patients observed at a dermatology clinic over an 18-month period.

Materials-The study included 321 patients with penile pathology. The term MBP was used to describe balanoposthitis of a localised, inflammatory nature with few, non-specific symptoms and a tendency to become chronic or recur. Two hundred and seventy had diseases clearly identifiable by clinical examination or laboratory tests; 51 cases were diagnosed as MBP and these patients had blood tests (to evaluate immune status) and microbiological examination; when these proved negative, a series of patch tests was also used.

Results-Of the 51 patients diagnosed as having MBP, the cause was ascertained in 34 cases (infection, mechanical trauma, contact irritation, contact allergy, etc.), whereas no specific aetiological factor was detected to explain the symptoms in the remaining 17 cases.
\end{abstract}

(Genitourin Med 1994;70:345-346)

\section{Introduction}

Diseases of the glans penis and/or prepuce are common in clinical practice and may involve: localised pathological signs, genital signs of dermatological disorders, balano-preputial signs of a disease originally developing in other organs.

This report summarises an 18-month experience of observation of mild balanoposthitis (MBP) (that is balanoposthitis of localised, inflammatory nature, with few, non-specific symptoms and a tendency to become chronic or recur).

Department of

Dermatology,

Department of

Urology, University of

Padua

C Veller Fornasa

A Calabrō

A Miglietta

M Tarantello

C Biasinutto

A Peserico

Address correspondence to: Dr C Veller Fornasa, Clinica Dermatologica Via Cesare Battisti, 206 35100 - Padova, Italy.

Accepted for publication 4 May 1994 and instrumental tests according to their presenting symptoms. In the case of syphiloma for example, the paraboloid microscope was used to test for Treponema pallidum and serological samples were taken to test for syphilis; microbiological investigations were performed in the case of balanoposthitis with a purulent exudate; biopsies were taken in the case of carcinoma, Queyrat's erythroplasia, melanoma and Zoon's balanitis; in the event of severe erythematous-exudative eczematous lesions of the penis, the Standard European series of patch tests ${ }^{1}$ was performed, adding the group of allergens typical of medications ${ }^{2}$ and substances contained in condoms. ${ }^{3}$ In particular, 51 patients presenting with MBP (age range 14-59, mean 34.3) underwent a complete blood count with a differential leukocyte count, blood glucose concentration and glucose tolerance curve, a complete urine test and urine culture, VDRL for syphilis and the TPHA test, Gerhardt's test for acetoacetic acid and lymphocyte sub-population typing. These patients' lesions were also tested by various suitable methods ${ }^{4-13}$ for any presence of Chlamydia trachomatis, Mycoplasma pneumoniae, Trichomonas vaginalis, Candida albicans, Neisseria gonorrhoeae, L-bacterial forms and aerobic bacterial flora, Haemophilus influenzae, Haemophilus ducreyi, $\beta$-haemolytic streptococci, and Ureaplasma urealyticum. An antibiogram was prepared on cases revealing micro-organisms. Material for microbiological investigations was obtained by scraping. Standard European-Series skin tests, adding allergens from the topical medication group and from substances contained in condoms, were performed on patients whose blood and/or microbiological tests proved negative. Given the mild clinical symptoms and the non-specificity of the histological examination of MBP, ${ }^{14}$ biopsies were not performed.

\section{Results}

Of the 321 patients observed, 185 were found to suffer from infectious diseases, 17 from traumatic/irritant contact balanoposthitis, three from severe erythematous-exudative allergic contact dermatitis (Balsam of Peru, Kathon CG and perfumes respectively), eight from neoplastic disease. There were also 11 cases of psoriasis, nine of lichen ruber planus, eight of lichen sclerosus et atrophicus and balanitis xerotica obliterans, five of aphthosis, four of penile glandular hypertrophy, three of pearly penile papules, three of vitiligo, three of Zoon's balanitis, three of erythema multiforme, three of fixed drug eruption, two of atopic dermatitis, one of annular granuloma, one of pemphigus and one of pemphigoid.

The other 51 patients were diagnosed as 
Table Ascertained causes of $M B P$

\begin{tabular}{lll}
\hline Causes & & Cases \\
\hline & Candida albicans & 4 \\
& Chlamydia trachomatis & 2 \\
B-haemolitic streptococcus & 2 \\
Infection & Candida albicans/Klebsiella & 1 \\
& Haemophilus parainfluenzae & 1 \\
& Klebsiella & 1 \\
& Enterococcus/Haemophilus & 1 \\
& parainfluentiae/ & \\
& Stafilococcus epidermidis/ & \\
Contact irritation & Escherichia coli & 9 \\
Mechanical trauma & & 7 \\
& sodium lauryl sulphate & 1 \\
& perfumes mix & 1 \\
Contact allergy & mercaptobenzothiazole & 1 \\
& mercaptobenzothiazole/ & 1 \\
& penicillamine/thiuram mix & 1 \\
& etylene diamine & \\
& nickel sulphate/kathon CG & 1 \\
\hline
\end{tabular}

having MBP. The table illustrates the causes of MBP ascertained in 34 out of 51 patients, namely infectious agents in 12 cases, mechanical trauma (rubbing, sexual intercourse, etc.) in seven cases, contact irritation in nine and contact sensitisation in six. After suitable treatment, all 34 patients achieved complete clinical cure. In particular, the 12 patients found with micro-organisms were cured with a specific antibiotic treatment.

No specific aetiological factor was detected to explain the symptoms in the remaining 17 patients, eight of whom nonetheless achieved clinical remission after using topical emollients or correcting their personal hygienic habits.

\section{Conclusions (table)}

MBP can be the mild clinical expression of an infectious disease, so it is of primary diagnostic importance to perform specific microbiological and immunological tests. In fact, our series revealed 12 cases in which the symptoms were mild, but were nonetheless secondary to infectious processes. It could be claimed that the finding of certain microorganisms does not necessarily mean that they are pathogenic. However, the fact that the 12 patients were cured by a specific antibiotic treatment would suggest that these microorganisms had a pathogenic as well as a saprophytic role.

The medical literature reports a number of cases of balanoposthitis due to sensitisation (to condoms, perfumes, detergents, etc.). ${ }^{915-17}$. Our study suggests that sensitisation to such topical agents tends more often to develop as MBP (six cases) than as an erythematous-exudative balanoposthitis (three cases).
As already mentioned, no aetiological explanation was found for the MBP in 17 out of 51 cases, which confirms that balanoposthitis may sometimes represent a frustrating problem for the clinician (as well as for the patient, of course)..$^{18}$ The finding that some of these 17 patients achieved clinical remission of symptoms after using topical emollients or correcting their personal hygiene routines coincides with other reports, ${ }^{19}$ confirming that balanoposthitis can sometimes be caused by non-specific factors such as retention of smegma and inadequate or unsuitable personal hygiene. There remains the matter of the psychological component which, in our opinion, should be considered as a possible cause, or contributory factor, in this type of pathology. ${ }^{20-22}$

1 Notice: Revised European Standard series. Contact Dermatitis 1988;19:391.

2 A Sertoli: Dermatologia Allergologica Professionale ed Ambientale. Roma, II Pensiero Scientifico Ed.: 1991, vol.2, 588-91.

3 A Sertoli: Dermatologia Allergologica Professionale ed Ambientale. Roma, II Pensiero Scientifico Ed.: 1991, vol.2, 612 .

4 Schachter J. Chlamydie. In: Balows A, Hauser WJ jr, Herrmann KL, Isenberg HD, Shadomy HJ eds. Manual of Clinical Microbiology. 5th ed. Washington. American Society for Microbiology. 1991

5 Graman PS, Menegus MA. Microbiology Laboratory tests. In: Reese RE, Douglas RG jr. A practical approach to Infectious Disease. 2nd ed. Boston. Little Brown and Company. 1986

6 Kreiger JN, Tam MR, Stevens CE, et al. Diagnosis of trichomoniasis. Comparison of conventional wet-mount examination with cytologic studies, cultures, and monoclonal antibody staining of direct specimens. $\mathcal{f} A M A$ 1988;259:1223-7.

7 Vidotto V. I lieviti in Micologia Medica. Milano, DID 1992.

8 Cavicchini S, Alessi E. Metodi di identificazione della Neisseria gonorrhoeae. G. Ital Dermatol Venereol 1987;122: $187-9$.

9 Vena GA, Montagna MT, Angelini G. Etiopathogenetic findings in balanoposthitis. G Ital Dermatol Venereol 1985;120:355-8.

10 Marschalko M, Vajda Z. Haemophilus influenzae in genital cellulitis and ulcer. Dermatol Monatsschr 1988;174: 634-7.

11 Ballard RC, Abeck D, Korting HC, Dangor Y, BraunFalco $O$. Morphologic variants of genital ulcer caused by Haemophilus ducreyi. Hautarzt 1989;40:443-7.

12 Kyriazi NC, Costenbader CL. Group A $\beta$-Hemolytic streptococcal balanitis: it may be more common than you think. Pediatrics 1991;88:154-6.

13 Hackel $H$, Hartmann AA, Elsner P, Burg G. Prevalence of Ureaplasma urealyticum in the urethra of man without urethritis in relation to clinical diagnosis. Dermatologica 1990;180:76-8.

14 Vohra S, Badlani G. Balanitis and balanoposthitis. Urol Clin North Am 1992;19:143-7.

15 Sonnex C. Sexual Hypersensitivity. Br f Hosp Med 1988; 39:40, 42, 47-8.

16 Fisher AA. Reactions of the mucous membrane to contactants. Clin Dermatol 1987;5:123-36.

17 Hindson TC. Studies in contact dermatitis. XVI. Contraceptives. Transactions St. Fohn's Hospital Dermatology Society 1966;52(1)

18 Brett MC, Coleman J. Common penile lesions. Off Urol 1988;15:671-85.

19 Ive FA, Wilkinson DS. Disease of the Umbilical, Perianal and Genital Regions. In: Rook A, Wilkinson DS, Ebling and Genital Regions. In: Rook A, Wilkinson DS, Ebling FJ, Champion RH, Burton JL. Textbook of Dermatology. 5th ed. London: Blackwell Scientific Publications 1992.
Koblenzer CS. Psychosomatic concepts in Dermatology. Arch Dermatol 1983;119:501-11.

21 Panconesi E. Lo Stress, le Emozioni e la Pelle. Milano, Masson 1989.

22 Bassi E. Introduzione alla Dermatologia Psicosomatica. Padova, Piccin 1977 